\title{
Manifestaciones neurológicas en la enfermedad del coronavirus 2019
}

\section{Teresita Corona, ${ }^{1,2}$ Mayela Rodríguez-Violante ${ }^{2,3}$ y Guillermo Delgado-García ${ }^{2,3 *}$}

${ }^{1}$ Academia Nacional de Medicina; ${ }^{2}$ Programa de Maestría y Doctorado en Ciencias Médicas, Odontológicas y de la Salud, Universidad Nacional Autónoma de México; 'aboratorio Clínico de Enfermedades Neurodegenerativas, Instituto Nacional de Neurología y Neurocirugía "Manuel Velasco Suárez". Ciudad de México, México

PALABRAS CLAVE: COVID-19. Coronavirus. Neurología. Manifestaciones neurológicas. Enfermedades del sistema nervioso.

\section{Neurological manifestations in coronavirus disease 2019}

\begin{abstract}
Coronavirus disease 2019 (COVID-19), an infection caused by severe acute respiratory syndrome coronavirus 2 (SARS-CoV-2), is currently hitting the world in the form of a pandemic. Given that some reports suggest that this infection can also occur with neurologic manifestations, this narrative review addresses the basic and clinical aspects concerning the nervous system involvement associated with this disease. More than one third of patients hospitalized for COVID-19 can present with both central and peripheral neurological manifestations. The former include dizziness and headache, while the latter include taste and smell disturbances. Other reported neurological manifestations are cerebrovascular disease and epileptic seizures. According to published reports, neurological disorders are not uncommon in COVID-19 and can sometimes represent the first manifestation of the disease; therefore, neurologists should consider this diagnostic possibility in their daily practice. Since maybe not all COVID-19 neurological manifestations are due to SARS-CoV-2 direct effects, it is important to monitor the rest of the clinical parameters such as, for example, oxygen saturation. Similarly, follow-up of patients is advisable, since whether neurological complications may develop lately is thus far unknown.
\end{abstract}

KEY WORDS: COVID-19. Coronavirus. Neurology. Neurological manifestations. Diseases of the nervous system.
Correspondencia:

*Guillermo Delgado-García

E-mail: guillermo.delgadogr@comunidad.unam.mx

0016-3813/@ 2020 Academia Nacional de Medicina de México, A.C. Publicado por Permanyer. Este es un artículo open access bajo la licencia

CC BY-NC-ND (http://creativecommons.org/licenses/by-nc-nd/4.0/).
Fecha de recepción: 14-04-2020

Fecha de aceptación: 12-05-2020

A.C. Publicado por Permanyer. Este es un artículo open access bajo la licencia

Gac Med Mex. 2020;156:317-320

Disponible en PubMed

\section{Resumen}

La enfermedad del coronavirus 2019 (COVID-19), infección causada por el coronavirus 2 del síndrome respiratorio agudo grave (SARS-CoV-2), actualmente afecta al mundo en forma de una pandemia. Debido a que algunos reportes apuntan a que esta infección puede cursar también con manifestaciones neurológicas, en esta revisión narrativa se abordan los aspectos básicos y clínicos concernientes a la afectación del sistema nervioso por esta enfermedad. Más de un tercio de los pacientes hospitalizados por COVID-19 pueden presentar manifestaciones neurológicas, tanto centrales como periféricas. Entre las primeras se encuentran el mareo y la cefalea; $y$ entre las segundas, las alteraciones del gusto y el olfato. Otras manifestaciones neurológicas reportadas son la enfermedad vascular cerebral y las crisis epilépticas. Según los informes publicados, los padecimientos neurológicos no son infrecuentes en COVID-19 y en ocasiones pueden representar la primera manifestación de la enfermedad, de modo que los neurólogos deberán considerar esta posibilidad diagnóstica en su práctica cotidiana. Dado que no todas las manifestaciones neurológicas de COVID-19 pudieran deberse a efectos directos de SARS-CoV-2, es importante monitorear el resto de los parámetros clínicos, por ejemplo, la oxigenación. De igual forma, es recomendable el seguimiento de los pacientes, ya que hasta el momento se ignora si las complicaciones neurológicas pueden desarrollarse tardíamente.
\end{abstract}




\section{Introducción}

La enfermedad del coronavirus 2019 (COVID-19, coronavirus disease 2019) es causada por el coronavirus 2 del síndrome respiratorio agudo grave (SARS-CoV-2, severe acute respiratory syndrome coronavirus 2), un virus envuelto de ácido ribonucleico monocatenario positivo. A finales de diciembre de 2019, un brote de neumonía de causa desconocida se presentó en Wuhan, China, y posteriormente se propagó con rapidez en ese país. Al poco tiempo se determinó que un nuevo coronavirus era el agente causal. ${ }^{1,2}$ Para el 13 de abril de 2020, el brote se había convertido en una pandemia que afectaba a 213 países, áreas o territorios en todo el mundo, con más de 1.8 millones de casos confirmados, entre los cuales 117217 (6.3 \%) tuvieron un desenlace fatal. ${ }^{3}$ En México se habían confirmado 5014 casos y 332 (6.6\%) defunciones por esta enfermedad. La entidad federativa con mayor número de casos positivos y defunciones era y es hasta el momento de este informe la Ciudad de México. ${ }^{4}$

EI SARS-CoV-2 se transmite principalmente a través de gotas respiratorias (de entre 5 y $10 \mu \mathrm{m}$ ) y por contacto. Este coronavirus también se ha detectado en materia fecal y lágrimas; su periodo de incubación es de cinco días en promedio, con un rango de uno a 14 días. Prácticamente todos los pacientes (95\%) presentan síntomas en los primeros 12.5 días después del contacto. No obstante, existen portadores asintomáticos, quienes constituyen una fuente potencial de infección. Los síntomas más comunes son fiebre, fatiga y tos no productiva, seguidos de cefalea, congestión nasal, odinofagia, mialgias y artralgias. ${ }^{1,2}$ Sin embargo, desde finales de febrero aparecieron los primeros reportes de pacientes con COVID-19 que cursaban con manifestaciones neurológicas. ${ }^{5,6} \mathrm{~A}$ esos reportes iniciales siguieron otros que al parecer apuntan en esta misma dirección. ${ }^{7}$ Debido a lo anterior, en esta revisión se abordan los aspectos básicos y clínicos concernientes a la afectación del sistema nervioso en esta enfermedad.

\section{Aspectos básicos}

Los coronavirus no son completamente desconocidos en neurología; se han empleado, por ejemplo, en el desarrollo de modelos murinos de enfermedades desmielinizantes. ${ }^{8}$ Existe evidencia que identifica el neurotropismo como una característica de los coronavirus. ${ }^{9}$
SARS-CoV-2 pertenece al género de los betacoronavirus (junto con SARS-CoV y MERS-CoV, entre otros) y actualmente se conoce la propensión de los betacoronavirus a la neuroinvasividad. ${ }^{9}$

Las primeras propuestas acerca de la potencial neuroinvasividad de SARS-CoV-2 fueron inferidas por analogía, considerando la evidencia obtenida de otros betacoronavirus, especialmente de SARS-CoV, ya que ambos comparten secuencias (79.5\%) y, además, utilizan el mismo receptor para ingresar en las células humanas, una proteína transmembranal llamada enzima convertidora de la angiotensina 2 (ACE2), ${ }^{9,10}$ la cual se expresa normalmente en el endotelio de la vasculatura cerebral..$^{11,12}$ La catepsina L, una endopeptidasa lisosomal ampliamente distribuida en el sistema nervioso central, es la mediadora en la interacción inicial entre el virus y la célula del hospedero. ${ }^{12}$

Otro aspecto relevante es la patofisiología tras la disfunción olfatoria en los pacientes con COVID-19, la cual al parecer no es exclusiva de SARS-CoV-2 y puede hallarse en infecciones por otros coronavirus, que se detectan en la descarga nasal de pacientes con disfunción olfatoria. Algunos de estos pacientes no recobraron la olfacción después de recuperarse de la infección, aun cuando los resultados de la rinometría acústica eran normales, lo cual podría indicar que la inflamación nasal y la obstrucción asociada no son los únicos factores subyacentes de la disfunción olfatoria en estas infecciones. ${ }^{13}$

Se ha sugerido la diseminación directa de SARS-CoV-2 a través del transporte neuronal retrógrado (olfatorio o vagal) o la circulación sistémica; ${ }^{11,12}$ la primera de estas dos hipótesis ya ha sido demostrada en otros coronavirus utilizando modelos murinos. ${ }^{14}$ En uno de esos modelos, después de atravesar el bulbo olfatorio, SARS-CoV se diseminó hacia zonas relacionadas anatómicamente con la vía olfatoria, incluyendo la corteza piriforme..$^{13}$

\section{Aspectos clínicos}

Más de un tercio (36.4\%) de los pacientes hospitalizados por COVID-19 puede presentar manifestaciones neurológicas, tanto centrales como periféricas. Entre las primeras se encuentra el mareo y la cefalea y entre las segundas, las alteraciones del gusto y el olfato. Las manifestaciones neurológicas son más frecuentes en los pacientes con neumonía grave, incluidos el infarto y las hemorragias cerebrales. En este contexto también pueden presentarse crisis epilépticas 
focales; ;,6 algunos reportes sugieren que las crisis epilépticas durante esta infección podrían tener una frecuencia mayor a la registrada en la literatura. ${ }^{15}$ Excepto la enfermedad vascular cerebral (EVC) y la alteración del estado de vigilia, la mayoría de los trastornos neurológicos suelen ocurrir tempranamente en el curso de la enfermedad, es decir, uno o dos días después del inicio de los síntomas 0 , incluso, es posible que constituyan las primeras manifestaciones. ${ }^{5,6}$

Las alteraciones del olfato y el gusto se presentan en la mayoría de los pacientes con COVID-19 leve a moderada ( 85.6 y $88.8 \%$, respectivamente); en uno de cada 10 pacientes, la disfunción olfatoria precede al resto de los síntomas. Estos trastornos se han reportado con mayor frecuencia en pacientes del sexo femenino. Las disfunciones específicas más frecuentes son la anosmia $(79.6 \%)$ y la hipogeusia $(78.9 \%)$. Al parecer, la disfunción olfatoria en el contexto de COVID-19 no se explica del todo por la obstrucción nasal o la rinorrea, como se ha observado en SARSCoV. En poco menos de una cuarta parte de los pacientes, la disfunción gustatoria ha sido reportada como fluctuante. Menos de la mitad (44\%) de los pacientes con COVID-19 leve a moderada recupera la olfacción en el periodo temprano. Por otra parte, también se ha observado disfunción gustatoria residual, aunque menos frecuentemente que la olfatoria..$^{13}$

En el espectro de la EVC, el infarto es la presentación más frecuente en pacientes con COVID-19; otras manifestaciones son la hemorragia y la trombosis venosa. ${ }^{7}$ Algunos autores sostienen que la hemorragia cerebral en pacientes con COVID-19 es más frecuente de lo que se ha reportado en la literatura. ${ }^{16} \mathrm{En}$ general, la edad (71.6 \pm 15.7 años), la gravedad y el riesgo cardiovascular son mayores en los pacientes con COVID-19 y EVC. Los factores de riesgo cardiovascular más comunes en estos pacientes son la hipertensión arterial sistémica, la diabetes mellitus y la historia de EVC. Un reporte preliminar indicó $38 \%$ de mortalidad en estos pacientes. ${ }^{7}$

En un artículo se indicó que la potencial neuroinvasividad de SARS-CoV-2 podría estar asociada a la insuficiencia respiratoria que puede presentarse por COVID-19; en ese mismo documento, los hallazgos en uno de los sobrevivientes ${ }^{9}$ podrían interpretarse como síndrome de Ondina, es decir, falla en el automatismo respiratorio durante el estado de vigilia.

Otras manifestaciones menos frecuentes son ataxia, ${ }^{5,6}$ mielitis $^{17}$ y polineuropatía desmielinizante inflamatoria aguda. ${ }^{18}$ A principios de mayo de 2020 , en Pekín se reportó por primera vez la presencia de SARS-CoV-2 en el líquido cefalorraquídeo (LCR) de un hombre de 56 años con síntomas compatibles con encefalitis. ${ }^{10,14} \mathrm{En}$ un caso de meningoencefalitis, reportado en Japón, el SARS-CoV-2 se detectó en el LCR, pero no en la muestra nasofaríngea.$^{19}$ Un caso de COVID-19 asociado a encefalopatía necrosante hemorrágica aguda fue reportado en Detroit, si bien no fue posible determinar la presencia de SARS-CoV-2 en el LCR. ${ }^{20}$

Los pacientes con manifestaciones neurológicas de origen central suelen también presentar linfopenia, trombocitopenia y azoemia. ${ }^{5,6}$ Es importante considerar que posiblemente no todas las afectaciones del SARS-CoV-2 al sistema nervioso sean directas y podrían estar mediadas por la hipoxia o la respuesta inmune. . $^{10,15}$

Aunque en diversas publicaciones se ha sugerido que los pacientes que fallecieron a causa de COVID-19 grave presentaban edema cerebral y degeneración neuronal parcial, en el análisis histopatológico del tejido obtenido durante la autopsia ${ }^{10}$ no se incluyó tejido cerebral. ${ }^{21}$ Reportes subsecuentes tampoco incluyeron este tejido. ${ }^{22}$ Debido a lo anterior, recientemente se ha propuesto la realización de un estudio neuropatológico estandarizado en pacientes con COVID-19. ${ }^{23}$

\section{Posibilidades terapéuticas}

Algunos autores han sugerido que COVID-19 debería tratarse tempranamente para reducir las complicaciones extrapulmonares, incluyendo las neurológicas;, ${ }^{24}$ sin embargo, esta recomendación no está basada en evidencia sólida. En Stroke and Vascular Neurology se publicó un consenso sobre la prevención y manejo de COVID-19 dirigido específicamente a los neurólogos. Entre las múltiples recomendaciones se sugiere tratar a los pacientes con infección leve con umifenovir o cloroquina (500 mg cada 12 horas por 10 días) durante los primeros días. ${ }^{25}$ Recientemente se ha discutido que el uso de cloroquina en China está asociado a un ensayo clínico multicéntrico ${ }^{26}$ cuyos resultados no están disponibles para su consulta. ${ }^{27}$ Actualmente, múltiples ensayos clínicos en China y otros países se encuentran reclutando pacientes para valorar el efecto y seguridad de la cloroquina en COVID-19. ${ }^{26-28}$ No hay que olvidar que la cloroquina puede asociarse a múltiples efectos adversos, incluyendo prolongación del segmento QT y arritmias ventriculares. ${ }^{28}$ Por otra parte, el umifenovir no está disponible en México.

En la actualidad, no se ha reportado trombólisis intravenosa ni trombectomía mecánica en pacientes 
con COVID-19, los cuales han sido tratados con antiagregantes (ácido acetilsalicílico o clopidogrel) o anticoagulantes (heparina de bajo peso molecular) cuando presentan EVC. ${ }^{7}$

\section{Conclusiones}

Los padecimientos neurológicos no son infrecuentes en COVID-19 y en ocasiones pueden representar la primera manifestación de la enfermedad; por ello, los neurólogos deben considerar esta posibilidad diagnóstica en su labor rutinaria. Como probablemente no todas las manifestaciones neurológicas de los pacientes de COVID-19 se deban a efectos directos de SARS-CoV-2, es importante monitorizar el resto de las variables clínicas de los pacientes, por ejemplo, la oxigenación.

\section{Agradecimientos}

Guillermo Delgado García agradece al Consejo Nacional de Ciencia y Tecnología por la beca nacional para cursar los estudios de maestría.

\section{Conflicto de intereses}

Los autores declaran no tener conflictos de intereses.

\section{Financiamiento}

Los autores no recibieron patrocinio para llevar a cabo este artículo.

\section{Responsabilidades éticas}

Protección de personas y animales. Los autores declaran que para esta investigación no se realizaron experimentos en seres humanos ni en animales.

Confidencialidad de los datos. Los autores declaran que en este artículo no aparecen datos de pacientes.

Derecho a la privacidad y consentimiento informado. Los autores declaran que en este artículo no aparecen datos de pacientes.

\section{Bibliografía}

1. Zhou M, Zhang X, Qu J. Coronavirus disease 2019 (COVID-19): A clinical update. Front Med. 2020;14:126-135.

2. He F, Deng Y, Li W. Coronavirus disease 2019: What we know? J Med Virol. 2020 Mar 24. DOI: 10.1002/jmv.25766
3. World Health Organization [Internet]. Suiza: Coronavirus disease (COVID-19) pandemic; 2020. Disponible en: https://www.who.int/emergencies/diseases/novel-coronavirus-2019

4. Secretaría de Salud [Internet]. México: Comunicado técnico diario nuevo coronavirus en el mundo (COVID-19), 13/04/2020; 2020. Disponible en: https://beta.slp.gob.mx/SSALUD/Documentos\%20compartidos/Coronavirus/abril/Comunicado_Tecnico_Diario_COVID-19_2020.04.13.pdf

5. Mao L, Wang M, Chen S, He Q, Chang J, Hong C, et al. Neurological manifestations of hospitalized patients with COVID-19 in Wuhan, China: A retrospective case series study. medRxiv. 2020 Feb 25. DOI: 10.1101/2020.02.22.20026500

6. Mao L, Jin H, Wang M, Hu Y, Chen S, He Q, et al. Neurologic manifestations of hospitalized patients with coronavirus disease 2019 in Wuhan, China. JAMA Neurol. 2020:e201127.

7. Li Y, Wang M, Zhou Y, Chang J, Xian Y, Mao L, et al. Acute cerebrovascular disease following COVID-19: A single center, retrospective, observational study. SSRN. 2020 Jan. DOI: 10.2139/ssrn.3550025

8. Libbey JE, Lane TE, Fujinami RS. Axonal pathology and demyelination in viral models of multiple sclerosis. Discov Med. 2014;18:79-89.

9. Li YC, Bai WZ, Hashikawa T. The neuroinvasive potential of SARS-CoV2 may play a role in the respiratory failure of COVID-19 patients $\mathrm{J}$ Med Virol. 2020 Mar 11. DOI: 10.1002/jmv.25728

10. Wu Y, Xu X, Chen Z, Duan J, Hashimoto K, Yang L, et al. Nervous system involvement after infection with COVID-19 and other coronaviruses. Brain Behav Immun. 2020 Jul;87:18-22. DOI: 10.1016/j.bbi.2020.03.031.

11. Baig AM, Khaleeg A, Ali U, Syeda H. Evidence of the COVID-19 virus targeting the CNS: Tissue distribution, host-virus interaction, and proposed neurotropic mechanisms. ACS Chem Neurosci. 2020;11:995-998.

12. Toljan K. Letter to the editor regarding the viewpoint "Evidence of the COVID-19 virus targeting the CNS: tissue distribution, host-virus interaction, and proposed neurotropic mechanism". ACS Chem Neurosci. 2020;11:1191-1194.

13. Lechien JR, Chiesa-Estomba CM, de Siati DR, Horoi M, le Bon SD, Rodríguez $\mathrm{A}$, et al. Olfactory and gustatory dysfunctions as a clinical presentation of mild-to-moderate forms of the coronavirus disease (COVID-19): A multicenter European study. Eur Arch Otorhinolaryngol. 2020:1-11.

14. Nath A. Neurologic complications of coronavirus infections. Neurology. 2020;94:809-810. DOI: 10.1212/WNL.0000000000009455.

15. Baig AM. Neurological manifestations in COVID-19 caused by SARSCoV-2. CNS Neurosci Ther. 2020;26:499-501.

16. Wang HY, Li XL, Yan ZR, Sun XP, Han J, Zhang BW. Potential neurological symptoms of COVID-19. Ther Adv Neurol Disord. 2020 Mar 28. DOI: 10.1177/1756286420917830

17. Zhao K, Huang J, Dai D, Feng Y, Liu L, Nie S. Acute myelitis after SARSCoV-2 infection: A case report. medRxiv. $2020 \mathrm{Apr} 09$. DOI: $10.1101 / 2020.03 .16 .20035105$

18. Zhao H, Shen D, Zhou H, Liu J, Chen S. Guillain-Barré syndrome associated with SARS-CoV-2 infection: causality or coincidence? Lancet Neurol. 2020;19:383-384.

19. Moriguchi T, Harii N, Goto J, Harada D, Sugawara H, Takamino J, et al. A first case of meningitis/encephalitis associated with SARS-coronavirus-2. Int J Infect Dis. 2020;94:55-58.

20. Poyiadji N, Shahin G, Noujaim D, Stone M, Patel S, Griffith B. CO VID-19-associated acute hemorrhagic necrotizing encephalopathy: CT and MRI features. Radiology. 2020 Mar 31;201187. DOI: 10.1148/radiol.2020201187.

21. Xu Z, Shi L, Wang Y, Zhang J, Huang L, Zhang C, et al. Pathological findings of COVID-19 associated with acute respiratory distress syndrome. Lancet Respir Med. 2020;8:420-422.

22. Tian S, Xiong Y, Liu H, Niu L, Guo J, Liao M, et al. Pathological study of the 2019 novel coronavirus disease (COVID-19) through post-mortem core biopsies. Mod Pathol. 2020;33:1007-1014. DOI: 10.1038/s41379020-0536-x

23. Cevik L, Alves M, Otero J. Neuropathologists play a key role in establishing the extent of COVID-19 in human patients. Free Neuropathology. 2020;1.11 Apr 02. DOI: 10.17879/freeneuropathology-2020-2736

24. Li Z, Huang Y, Guo X. The brain, another potential target organ, needs early protection from SARS-CoV-2 neuroinvasion. Sci China Life Sci. 2020 Mar 31:1-3. DOI: 10.1007/s11427-020-1690-y

25. Jin H, Hong C, Chen S, Zhou Y, Wang Y, Mao L, et al. Consensus for prevention and management of coronavirus disease 2019 (COVID-19) for neurologists. Stroke Vasc Neurol. 2020 Apr 1. DOI: 10.1136/svn2020-000382.

26. Gao J, Tian Z, Yang X. Breakthrough: Chloroquine phosphate has shown apparent efficacy in treatment of COVID-19 associated pneumonia in clinical studies. Biosci Trends. 2020;14:72-73.

27. Cortegiani A, Ingoglia G, Ippolito M, Giarratano A, Einav S. A systematic review on the efficacy and safety of chloroquine for the treatment of COVID-19. J Crit Care. 2020;57:279-283.

28. Yazdany J, Kim AHJ. Use of hydroxychloroquine and chloroquine during the COVID-19 pandemic: What every clinician should know. Ann Intern Med. 2020;172:754-755. 